\title{
Influencia auditiva de la pared posterosuperior del conducto auditivo externo, en la hipoacusia de conducción postimpanomastoidectomía
}

\author{
Hearing influence of the posterior ear canal wall, \\ in the conductive hearing loss after tympanomastoidectomy \\ Pedro Cortez V1', Fabián Rubio $\mathrm{M}^{2}$, Carlos Stott $\mathbf{C}^{1}$.
}

\begin{abstract}
RESUMEN
Introducción: El tratamiento de la otitis media crónica (OMC) colesteatomatosa es quirúrgico, cuyo objetivo es erradicar la enfermedad, evitar complicaciones y prevenir recurrencias. El gold standard es la timpanomastoidectomía con canal wall down (TCWD). Estudios en cadáver han definido que TCWD disminuye la audición 1-5 dB en frecuencias $<1.000 \mathrm{~Hz}$ y 0-10 dB entre 1.000 y $3.000 \mathrm{~Hz}$. De aquí nuestro interés por definir la influencia acústica de la pared posterosuperior del conducto auditivo externo (CAE).

Objetivo: Determinar en cuantos decibeles se corrige la audición al reconstituir pared posterior del CAE pos-TCWD.

Material y método: Trabajo experimental. A pacientes pos-TCWD se reconstituye de manera transitoria la pared posterior del CAE, realizándose audiometría pre y posprocedimiento.

Resultados: 23 pacientes (25 oídos), promedio 48,8 años (18-72 años). En 96\% existió una diferencia favorable al reconstruir la pared posterior, presentando una mejoría auditiva entre 1,2 y 10,6 dB $(4,2 \pm 2,8 \mathrm{~dB})$. En frecuencias $<1.000 \mathrm{~Hz}$ la mejoría fue de 6,0 $d B(p<0,001)$, entre 1.000-3.000 Hz fue 2,6 dB $(p<0,001)$ y >3.000 Hz no hubo diferencia. Considerando PTP de vía aérea la mejoría fue 4,6 dB ( $p<0,001)$.

Conclusión: Nuestro estudio demuestra que existe mejoría auditiva en la gran mayoría de oídos evaluados al reconstituir la pared posterosuperior del CAE, alcanzando 6 $d B$ en frecuencias $<1.000 \mathrm{~Hz}$ y 2,6 $d B$ en frecuencias medias. Si consideramos los PTP de vía aérea la mejoría es de 4,6 dB en presencia de pared posterior.
\end{abstract}

Palabras clave: Colesteatoma, timpanomastoidectomía, ganancia auditiva, canal auditivo.

\begin{abstract}
Introduction: Cholesteatomas treatment is surgical and the goals are complete resection of it, to prevent complications and recurrences. The gold standard operative technique is canal wall down tympanomastoidectomy (CWDT), which reduces the recu-
\end{abstract}

1 Otorrinolaringólogo, Servicio de Otorrinolaringología, Hospital Clínico Universidad de Chile, Santiago, Chile.

2 Otorrinolaringólogo, Servicio de Otorrinolaringología, Hospital La Florida, Santiago, Chile.

Recibido el 8 de abril, 2018. Aceptado el 29 de mayo, 2018. 
rrence rate lower than $2 \%$. Studies on human temporal bones have defined that CWDT causes a decrease of 1 to $5 \mathrm{~dB}$ at frequencies below $1000 \mathrm{~Hz}$ and 0 to $10 \mathrm{~dB}$ between 1000 and $3000 \mathrm{~Hz}$.

Aim: To determine how many decibels the hearing is improved by reconstituting the posterior wall of the ear canal (EC) in patients after CWDT.

Material and method: Experimental study. In patients after CWDT, the posterior wall of EC was reconstructed temporarily. Audiometry was performed before and after the procedure.

Results: 23 patients were enrolled (25 ears evaluated). Average age 48.8 years (range 18 to 72 years). In 96\% of the ears there was a difference after the procedure with a hearing improvement of $4.2 \pm 2.8 \mathrm{~dB}$. In frequencies below $1000 \mathrm{~Hz}$, hearing improvement was $6.0 \mathrm{~dB}(p<0.001)$, between $1000-3000 \mathrm{~Hz}$, the improvement was $2.6 \mathrm{~dB}$ $(p<0.001)$ and $>3000 \mathrm{~Hz}$ there was no difference. When considering the airway-conduction pure tone average (PTA), the difference was a $4.6 \mathrm{~dB}$ improvement $(p<0.001)$.

Conclusions: Our study shows that there is a hearing improvement in the vast majority of ears that were evaluated by reconstituting the posterior wall of the EC, reaching a gain of $6 \mathrm{~dB}$ at frequencies $<1000 \mathrm{~Hz}$ and 2,6 $\mathrm{dB}$ at mid frequencies. Considering the airway PTA, the improvement is $4.6 \mathrm{~dB}$ in the presence of posterior canal wall.

Key words: Cholesteatoma, tympanomastoidectomy, hearing improvement, ear canal.

\section{INTRODUCCIÓN}

El colesteatoma es un acúmulo anormal de epitelio escamoso queratinizado en un sitio anómalo del oído, pudiendo localizarse en oído medio, epitímpano, mastoides o ápex petroso ${ }^{1}$. Histológicamente se reconoce como un quiste benigno de células escamosas que consta de tres componentes: el contenido, la matriz y la perimatriz o lámina propia. El contenido es el componente central y está formado por escamas de queratina completamente diferenciadas, acelulares. La matriz es el componente intermedio y está formada por epitelio escamoso queratinizado y da sostén a la estructura quística. La perimatriz está formada por tejido de granulación y puede o no tener cristales de colesterol. Está en contacto con tejido óseo y es esta perimatriz la que produce las enzimas proteolíticas que pueden provocar destrucción ósea².

Aunque puede cursar en forma asintomática, muchos colesteatomas se manifiestan clínicamente como hipoacusia y otorrea. Si la enfermedad progresa puede llevar a la parálisis facial, disfunción vestibular, e incluso complicaciones intracraneales como abscesos cerebrales y meningitis entre otras, siendo todas estas manifestaciones debidas en gran parte a su capacidad de destruir hueso. Una vez establecido el colesteatoma su único tratamiento es la erradicación quirúrgica y posterior intento de reconstrucción del oído ${ }^{3}$. En términos generales este cuadro es poco frecuente, afectando a la población entre 3 a 70 años de edad, con una prevalencia estimada de 3/100.000 en población pediátrica y 9/100.000 en adultos ${ }^{1}$.

El tratamiento definitivo es únicamente quirúrgico, donde el objetivo principal es lograr la erradicación de la enfermedad, evitar complicaciones y prevenir recurrencias. En forma secundaria, se intenta mantener o mejorar la capacidad auditiva del paciente por medio de este procedimiento. Existen diversas técnicas quirúrgicas, tales como la aticoantrostomía (AAT) con antroexclusión o como la aticoantromastoidectomía (AAM), conocida como cirugía radical de oído, la que puede realizarse con preservación de la pared posterosuperior del conducto auditivo externo (canal wall up - CWU) 0 sin preservación de ésta (canal wall down - CWD) ${ }^{4}$.

El gold standard para el manejo de la otitis media crónica colesteatomatosa ha sido por años la cirugía radical con canal wall down $n^{5,6}$. La remoción de la pared posterosuperior del canal auditivo externo (CAE) permite la exposición completa del epitímpano y del oído medio, ayudando de esta forma a erradicar de forma completa la enfermedad, logrando disminuir la recidiva del colesteatoma a 
tasas inferiores al $2 \%{ }^{5}$. En cambio con la técnica de conservación de la pared posterosuperior del CAE preserva en cierta forma la anatomía y fisiología del oído medio, pero se describen tasas de recidiva de enfermedad de hasta el $36 \%$ en adultos y $67 \%$ en niños 6 . Por otra parte, las modificaciones que se realizan en el oído medio a través de estos procedimientos, repercuten de forma secundaria en la transmisión del sonido desde la membrana timpánica a la cóclea. Es difícil identificar y aislar en un contexto clínico, los efectos acústicos de las modificaciones quirúrgicas realizadas en estos pacientes respecto de los efectos de la patología coexistente del oído medio. De tal manera, algunos estudios con modelos en cadáveres han intentado definir la repercusión acústica de las modificaciones realizadas en cirugía radial de oído con técnica CWU versus CWD. Whittemore y cols $^{5}$, describen que en sus mediciones de impedancia en hueso temporal de cadáver, la mastoidectomía CWD produce una disminución de 1-5 dB en las frecuencias por debajo de $1.000 \mathrm{~Hz}$ y aumenta en 0-10 dB entre los 1.000 y $3.000 \mathrm{~Hz}$, no habiendo diferencias significativas por sobre los $3.000 \mathrm{~Hz}$.

Otros autores han definido que la audición final es 5-10 dB peor en pacientes sometidos a timpanomastoidectomía con técnica CWD respecto de Ios pacientes con $\mathrm{CWU}^{6}$. Tanto por intentar mejorar las condiciones de la cavidad radical como para obtener mejores resultados funcionales, muchos cirujanos realizan la obliteración mastoidea habitualmente con injerto de hueso molido 0 fascia ${ }^{6,7}$.
En 2012, Woo Cho y cols ${ }^{8}$, publican los resultados de su estudio experimental en la que la obliteración mastoidea la realizan con injerto de silicona, logrando mejorar la conducción ósea en $12 \mathrm{~dB}$ y la conducción aérea en $16 \mathrm{~dB}$ promedio. Recientemente, otro estudio realizado por Ezzat ${ }^{9}$ también demuestra significativa mejoría auditiva al realizar obliteración y relleno de mastoides durante una cirugía radical de oído con CWD tanto con material natural como sintético.

Con estos antecedentes nace nuestro interés por identificar la influencia de la pared posterosuperior en la hipoacusia de conducción secundaria a la aticoantromastoidectomía con técnica CWD, dado que hasta el momento solo ha sido demostrado en hueso temporal de cadáver humano y en otros estudios los resultados al reconstruir la pared posterior están mezclados con el tiempo funcional que se ofrece en la misma cirugía radical de oído.

\section{MATERIAL Y MÉTODO}

\section{Diseño de estudio: Trabajo experimental}

La reconstitución de la pared posterior del CAE se realizó de forma transitoria utilizando ear plug de silicona moldeable (Figura 1), la cual se adaptó a la cavidad radical para reconstituir la pared posterior del conducto. Dicho procedimiento se realizó, previo consentimiento informado, en box de

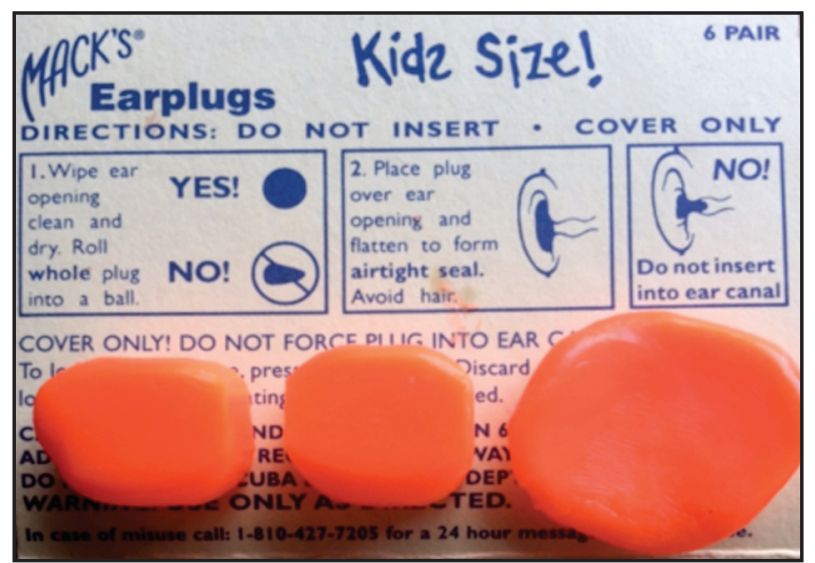

Figura 1. Silicona moldeable para la reconstitución transitoria de pared posterior de CAE. 
procedimientos del servicio de otorrinolaringología, bajo visión microscópica y por médicos del mismo servicio y autores de este trabajo. A todos estos pacientes se les realizó una audiometría antes y después de realizar la aplicación del ear plug de silicona en su sitio. El presente trabajo cuenta con la aprobación del Comité de Ética del Hospital Clínico de la Universidad de Chile.

Fueron incluidos pacientes mayores de 18 años, con antecedente de cirugía radical de oído con técnica abierta (CWD) y que acudieron a control durante los años 2014 a 2015. La cirugía debía ser al menos 6 meses antes del ingreso al estudio y los pacientes ingresados presentaron un gap aéreo/óseo $>20 \mathrm{~dB}$. Para realizar el procedimiento debían presentar una meatoplastía amplia. A todos los pacientes controlados por cirugía radical de oído con técnica abierta se les aplicó una encuesta de ingreso que consignaba datos biodemográficos y fueron evaluados con otomicroscopía y audiometría basal. Aquellos que cumplían con los criterios de ingreso se les solicitó el consentimiento informado y se les realizó el procedimiento, aplicándoles el ear plug de silicona de acuerdo a lo descrito. Posterior a ello se les realizó una nueva audiometría. Finalizada la segunda audiometría, el ear plug de silicona fue removido en su totalidad.

\section{Análisis estadístico}

Se utilizaron descriptores estadísticos generales, como media y desviación estándar para variables continúas de distribución normal y porcentajes para variables cualitativas. Se realizó comparación de variables continuas con T-student. Se consideró una significancia con valor $p<0,05$. Para el análisis se utilizó el software STATA 12.0 (StataCorp, College Station, Texas 77845-4512, USA).

\section{RESULTADOS}

Durante el período de estudio fueron ingresados 23 pacientes que cumplían los criterios de inclusión y exclusión, siendo 25 los oídos evaluados. De los pacientes ingresados 11 eran de sexo femenino y 12 de sexo masculino, con una edad promedio de 48,8 años y un rango entre los 18 y 72 años. El tiempo promedio entre la cirugía y el ingreso al estudio fue de 11,37 \pm 11 años (Tabla 1).

En nuestro estudio se pudo observar que en el 96\% de los oídos evaluados existió una diferencia favorable al reconstruir la pared posterior, siendo ésta determinada con la disminución del gap aéreo óseo tras el procedimiento (Figura 2). Es decir, a excepción de un oído, todos presentaron una

Tabla 1. Características de la muestra estudiada

\begin{tabular}{|ll|}
\hline \multicolumn{2}{|l|}{ Características } \\
\hline$N$ & 25 oídos (23 pacientes) \\
\hline Edad & $48,88 \pm 16,84$ \\
Rango & $18-72$ años \\
Tipo desde cirugía & 11,37 años \pm 11 \\
OD/=0l & $14 / 11$ \\
\hline
\end{tabular}

OD: oído derecho, Ol: oído izquierdo.

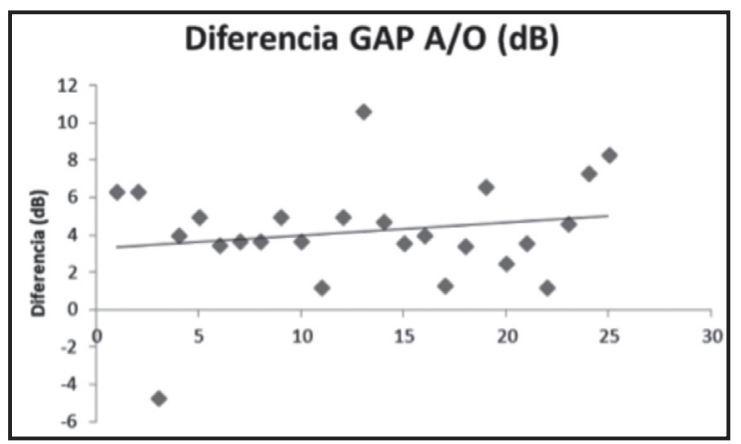

Figura 2. Diferencia de gap aéreo/óseo de los PTP. Decibeles de diferencia entre el PTP de vía aérea y PTP de vía ósea antes y después de la aplicación de la silicona, siendo en promedio de 4,2 dB de mejoría auditiva. 
mejoría auditiva de entre 1,2 y $10,6 \mathrm{~dB}$, siendo en promedio 4,2 $\pm 2,8 \mathrm{~dB}$ de mejoría auditiva al realizar la reconstitución de la pared posterior del CAE.

Los valores de los promedio tonal puro (PTP) y promedios de audición según frecuencias al ingreso y luego de la reconstrucción de la pared posterior del CAE se expresan en la Figura 3.

En la Figura 4 se observa, que al realizar el análisis por grupo de frecuencias, en el rango de frecuencia menores a $1.000 \mathrm{~Hz}$ la mejoría auditiva fue de $6,0 \mathrm{~dB}$ en promedio $(p<0,001)$. Para el grupo de frecuencia entre $1.000-3.000 \mathrm{~Hz}$ la mejoría fue de $2,6 \mathrm{~dB}(\mathrm{p}<0,001)$. En las frecuencias $>3.000 \mathrm{~Hz}$, la diferencia no fue estadísticamente significativa. Al considerar solo el PTP de vía aérea la diferencia fue de $4,6 \mathrm{~dB}$ de mejoría $(p<0,001)$ tras reconstituir la pared posterior.
Al realizar este mismo análisis de acuerdo al tipo de cirugía realizada encontramos que en el grupo de pacientes en que se realizó aticoantromastoidectomía (AAM) clásica (56\% de los pacientes) presentaban mayor ganancia auditiva $(4,92$ $\mathrm{dB}$ ) tras la restitución de la pared posterior del CAE respecto del grupo en que se les realizó AAM con algún tipo de relleno de cavidad mastoidea (fascia, paté de hueso) y respecto del grupo en que presentaba solo aticoantrostomía (AA). Pero esta diferencia, según el análisis de varianza de ANOVA no fue estadísticamente significativa (Figura 4).

Al consultar a los pacientes respecto del beneficio percibido durante la realización de la audiometría tras la reconstrucción de la pared posterior del CAE, éstos relataron una mejoría subjetiva según la escala visual análoga que resultó ser

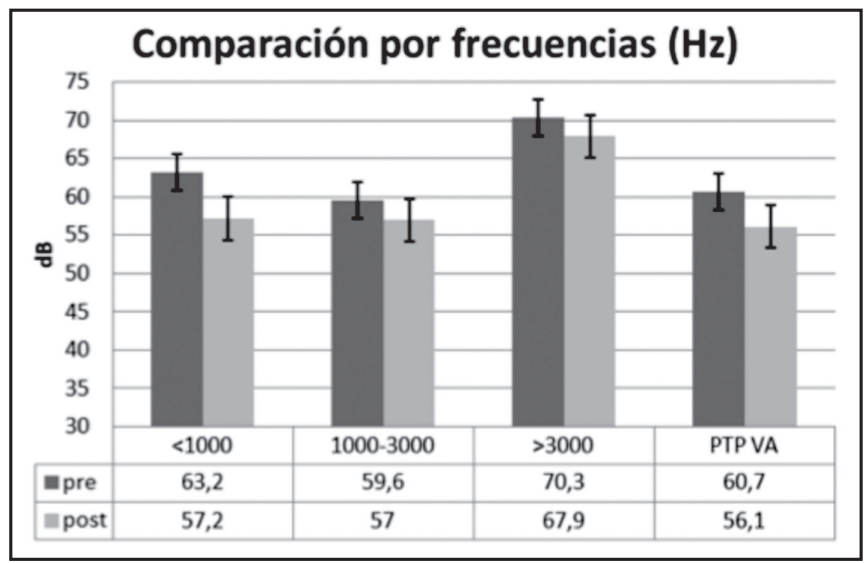

Figura 3. Comparación por frecuencias $(\mathrm{Hz})$ al ingreso y posreconstrucción de CAE con silicona. PTP VA: Promedio tonal puro de vía aérea.

Figura 4. Ganancia auditiva (dB) según diferencia de gap aéreo óseo y tipo de cirugía realizada. análisis de varianza (ANOVA $p=0,082$ ). AAM: aticoantromastoidectomía, AAM con $\mathrm{R}$ : aticoantromastoidectomía con relleno de mastoides, AA: aticoantrostomía.

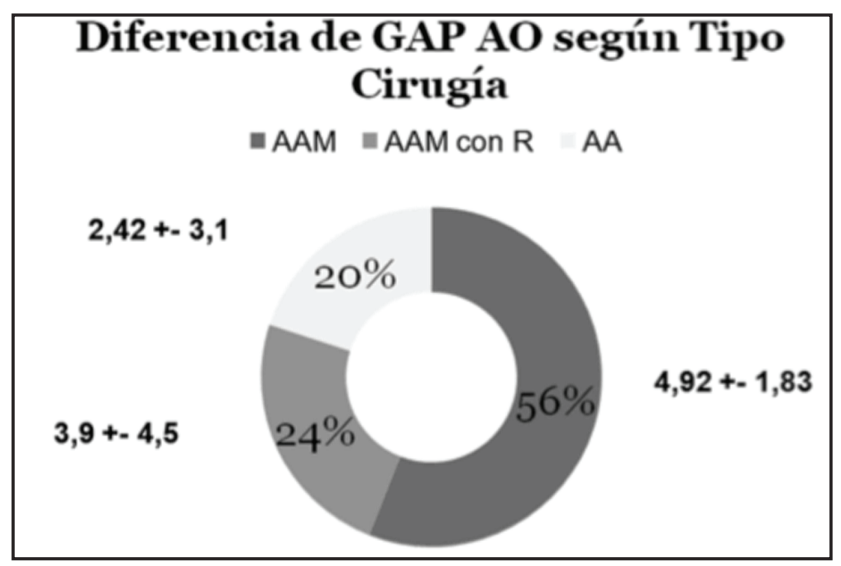


significativa estadísticamente. A pesar de que un tercio de los pacientes no notó esta diferencia subjetiva (Figura 5).

\section{DISCUSIÓN}

Hasta el día de hoy se discute respecto de cuál es la técnica quirúrgica ideal para enfrentar el colesteatoma de oído medio, teniendo cada una de estas técnicas, beneficios y desventajas. Una de las principales ventajas de la realización de la aticoantromastoidectomía clásica con rebaje de la pared posterior del CAE (canal wall down) es la disminución de la recidiva del colesteatoma, reduciendo en 3 veces el riesgo de que esto ocurra ${ }^{10}$.

Hasta el momento, solo un par de estudios de la literatura internacional, han demostrado cuál es la influencia auditiva de la pared posterior del CAE. Destaca el realizado por Whittemore ${ }^{7}$ en hueso temporal de cadáver humano el año 1998 en donde estableció que la preservación de la pared posterior del CAE tiene una influencia de entre 1 y $5 \mathrm{~dB}$ en las frecuencias menores de $1.000 \mathrm{~Hz}$ y de entre 0 y $10 \mathrm{~dB}$ para las frecuencias medias (entre 1.000 y $3.000 \mathrm{~Hz}$ ), no encontrando diferencia sobre los $3.000 \mathrm{~Hz}$. Nuestro estudio es el primero que se realiza en paciente vivo, y los resultados no son muy distintos de los encontrados por Whittemore ${ }^{7}$ y Gyo y cols ${ }^{11}$. Eso sí, nuestro estudio demuestra que existe una mejoría auditiva principalmente en las frecuencias bajas $(<1.000 \mathrm{~Hz})$ que alcanza los $6 \mathrm{~dB}$ y que esta sería menos significativa en las frecuencias medias (1.000-3.000 Hz) siendo de 2,6 $\mathrm{dB}$ en promedio al reconstituir la pared posterior del CAE. Así como en estudios previos, no se encontró diferencia en las frecuencias agudas sobre los $3.000 \mathrm{~Hz}$. Si consideramos los PTP de vía aérea la diferencia alcanzada es de 4,6 dB de ganancia auditiva al reconstituir la pared posterior del CAE.

Lo llamativo del estudio es que el $96 \%$ de los pacientes presentan algún grado de mejoría auditiva al reconstituir la pared posterior del CAE, encontrándose una ganancia auditiva de hasta 10,6 dB y en el $40 \%$ de estos pacientes las diferencia fue mayor a $5 \mathrm{~dB}$ de ganancia auditiva considerando el gap aéreo-óseo. Si consideramos solo las frecuencias bajas encontramos que sobre el $70 \%$ de los oídos evaluados presentaron una mejoría mayor de $5 \mathrm{~dB}$ tras el procedimiento.

Al evaluar la discriminación auditiva pre y posreconstitución de pared posterior, ésta no mostró diferencias significativas. Por otra parte, si analizamos la diferencia según tipo de cirugía realizada se observó que en el grupo de pacientes que tenían como antecedente quirúrgico la aticoantromastoidectomía clásica (con una cavidad radical amplia) se veían mayormente beneficiados de la reconstrucción de la pared posterior, respecto del grupo con cavidad mastoidea más pequeña (4,92 vs 3,9 $\mathrm{dB}$ ), pero esta diferencia no fue estadísticamente significativa.

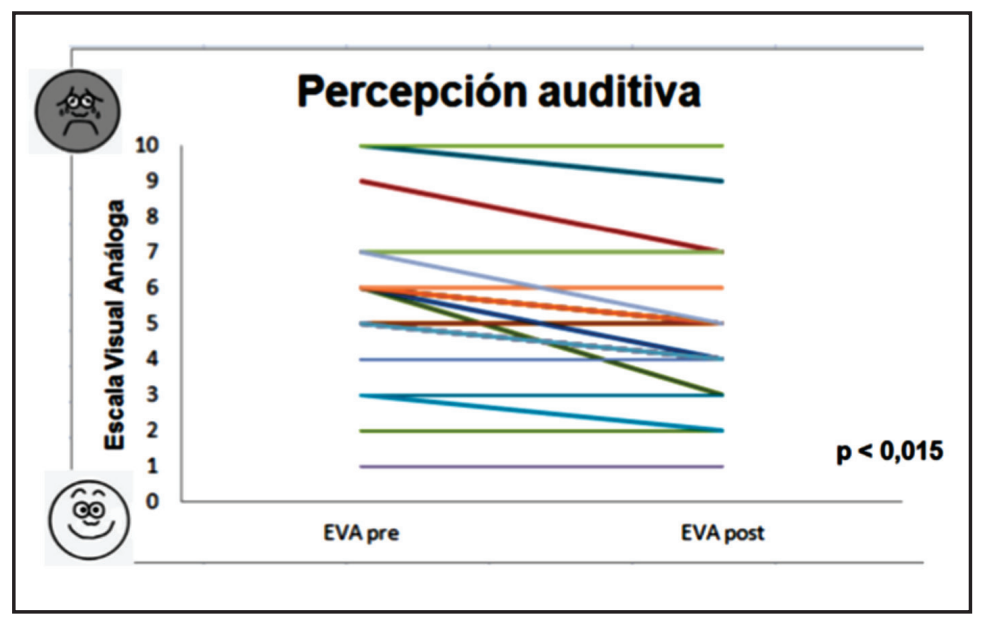

Figura 5. Percepción de mejoría auditiva subjetiva según aplicación de escala visual análoga, antes y después de la reconstitución de la pared posterior del CAE. 
Por último, si bien gran parte de los pacientes refieren haber sentido mejoría subjetiva según escala visual análoga, mejorando de 5,6 puntos a 4,8 puntos en promedio, creemos que esta diferencia no es clínicamente significativa, a pesar de serlo del punto de vista estadístico.

\section{CONCLUSIÓN}

Este trabajo es el primer estudio de este tipo que logra demostrar la influencia auditiva de la pared posterior del CAE en paciente vivo. Hasta el momento, esto solo había sido determinado en hueso temporal de cadáver y en otras series había solo estimaciones en pacientes operados, pero en los que no se podía separar sus resultados respecto de otras intervenciones que se le realizaban en el mismo acto.

Si bien estos resultados no van a influir en la toma de decisión del tipo de técnica a utilizar al enfrentar un colesteatoma de oído medio (CWD o CWU), cree-

\section{BIBLIOGRAFÍA}

1. Olszewska E, Wagner M, Bernal-Sprekelsen M et AL. Etiopathogenesis of cholesteatoma. Eur Arch Otorhinolaryngol 2004; 261: 6-24.

2. Cohen M, Callejas C, Salgado M. Fisiopatología del colesteatoma originado a partir de un bolsillo de retracción. Rev Otorrinolaringol Cir Cabeza Cuello 2006; 66: 39-46.

3. Stott C, Ortega G, Bravo G, Délano PH. Cirugía del colesteatoma y audición. Rev Otorrinolaringol Cir Cabeza Cuello 2013; 73: 243-8.

4. Laco J, Infante J, Pisón F. Consideraciones sobre la cirugía funcional del colesteatoma. Técnicas cerradas. Acta Otorrinolaring Esp 1997; 48: 115-20.

5. Whittemore K, Merchant S, Rosowsk J. Acoustic mechanisms: Canal wall-up versus canal walldown mastoidectomy. Otolaryngol Head Neck Surgery 1998; 118: 751-61.

6. KIm CW, OH JI, Cho KY, et AL. A technique for concurrent procedure of mastoid obliteration mos que sí puede influir en la opción de ofrecer relleno de la cavidad mastoidea y reconstrucción de pared posterior del canal auditivo externo siempre que sea posible. Esto, dado que ya conocemos que podríamos llegar a ganar hasta $10 \mathrm{~dB}$ de audición al tener pared posterior presente respecto que si no la tuviera. Ahora bien, la pérdida auditiva residual posoperatoria claramente hará necesario la indicación de audífono para lograr tener una audición funcional, lo cual no es corregido por el relleno de cavidad mastoidea ni reconstrucción de pared posterior del canal auditivo externo, pero tal vez esos $6 \mathrm{~dB}$ de ganancia auditiva puedan ser significativos para el paciente y el rendimiento del audífono implementado.

\section{Agradecimientos}

A equipo de tecnólogos médicos del Servicio de Otorrinolaringología del Hospital Clínico de la Universidad de Chile: Diego Olmedo, Rubén Olmedo y Cristián Martínez.

and meatoplasty after canal wall down mastoidectomy. Auris Nasus Larynx 2012; 39: 557-61.

7. Metha R, Harris J. Mastoid Obliteration. Otolaryngol Clin N Am 2006; 39: 1129-42.

8. Woо S, Сно YB, Сно HH. Mastoid Obliteration with Silicone Blocks after Canal Wall Down Mastoidectomy. Clinical and Experimental Otorhinolaryngology 2012; 5: 23-7.

9. Ezzat A, El-Begermy M, Mahmoud A, et al. Comparison Of Natural And Synthetic Materials To Improve Hearing After Obliteration Of The Mastoid Cavity. J Hear Sci 2015; 4: 36-41.

10. Tomlin J, Chang D, McCutcheon B, et al. Surgical Technique and recurrence in Cholesteatoma: a meta-analysis. Audiol Neurotol 2013; 18 : 135-42.

11. Gyo K, Goode RL, Miller C. Effect of middle ear modification on umbo vibration. Human temporal bone experiments with a new vibration measuring system. Arch Otolaringol Head Neck Surg 1986; 112: 1262-8. 